\title{
Degradação Redutiva de AZO-CORANTES UTILIZANDO-SE FERRO METÁLICO
}

\section{REDUCTIVE DEGRADATION OF AZO-DYES BY METALLIC IRON}

\begin{abstract}
CLÁUDIO LIMA DE SOUZA
Formado em Química pela Universidade Federal do Paraná, onde atualmente realiza curso de mestrado na área de remediação de resíduos de relevância ambiental.
\end{abstract}

\begin{abstract}
Patricio Peralta-Zamora
Professor, Doutor do Departamento de Química da Universidade Federal do Paraná, onde coordena o grupo de desenvolvimento de técnicas avançadas para tratamento de resíduos (tecnotrater)
\end{abstract}

\section{Recebido: 21/01/05 Aceito: 23/12/05}

\section{RESUMO}

Corantes azo são extensivamente utilizados em processos de tingimento de fibras têxteis, sendo caracterizados por elevada resistência frente a processos aeróbios de biodegradação e, por conseqüência, persistência nos processos convencionais de tratamento de resíduos. Neste trabalho reporta-se a degradação redutiva de corantes azo, utilizando-se ferro metálico. Em condições experimentais otimizadas ( $\mathrm{pH} 7$ e $10 \mathrm{~g}$ de lã de aço comercial) a completa descoloração do corante modelo (preto reativo 5) foi conseguida em um sistema contínuo, operando com tempos de retenção de $6 \mathrm{~min}$. Nestas condições, o ferro solubilizado alcança concentrações compatíveis com os limites impostos pela atual legislação brasileira $\left(12 \mathrm{mg} \mathrm{L}^{-1}\right)$. Trata-se de um resultado bastante promissor, principalmente levando-se em consideração o caráter recalcitrante dos azo corantes e a simplicidade do sistema proposto.

PALAVRAS-CHAVE: Ferro zero valente, azo corantes, descoloração, tratamento.

\section{ABSTRACT}

Azo dyes are extensively used in textile dying processes and are characterized by extreme resistance to biodegradation and consequently persistence during conventional wastewater treatment processes. In this work the reductive degradation of azo dyes was studied using zero-valent iron. At optimized experimental conditions ( $\mathrm{pH} 7$ and $10 \mathrm{~g}$ of commercial iron wool) complete decolorization of the model dye (reactive black 5) was afforded in a continuous system operating with hydraulic retention time of 6 min. At these conditions the released total soluble iron reaches a concentration compatible with the limits imposed by the current Brazilian legislation $\left(12 \mathrm{mg} \mathrm{L}^{-1}\right)$. That is a very promising result, mainly taking into account the high recalcitrant character of azo dyes and the simplicity of the proposed system.

KEYWORDS: Zero-valent iron, azo-dyes, decolorization, treatment.

\section{INTRODUÇÃO}

Embora a industria têxtil brasileira desempenhe um papel de inquestionável importância, destacando-se entre as principais atividades econômicas do país (Toledo, 2003), existem argumentos que fazem com que a sua compatibilidade com as novas diretrizes ambientais seja continuamente questionada, principalmente em função do elevado impacto associado aos resíduos líquidos produzidos em operaçóes de tingimento. Mesmo utilizando-se corantes de elevada capacidade de fixação, admite-se que, em média, 20\% de toda a carga de corantes utilizada em operações de tingimento é perdida nos resíduos do processo (Guaratini \& Zanoni, 2000). Como um agente complicador adicional, muitos destes corantes são resistentes ao tratamento biológico convencional (Perey et al, 2002), o que faz com que a sua presença seja constante nos efluentes das estaçōes de tratamento. Além do potencial poluente que resulta da introdução de cor no meio ambiente, preocupa a massiva utilização de corantes da família azo, os quais podem levar à geração de subprodutos de reconhecido caráter carcinogênico (Guaratini \& Zanoni, 2000).

Em função desta problemática, novas tecnologias têm sido recentemente propostas para a degradação de resíduos contendo corantes do tipo azo. Dentro deste contexto, destacam-se os trabalhos que reportam degradação redutiva, mediada por ferro metálico. Trata-se de um sistema bastante promissor, em função do custo relativamente baixo do ferro metálico e da sua elevada efetividade na de- gradação de contaminantes ambientais (Deng et al, 2000). O par redox $\mathrm{Fe}^{0} / \mathrm{Fe}^{2+}$ apresenta um potencial de oxidação de 0,44 V (Atkins, 2001), suficiente para promover a redução de inúmeros substratos de interesse (Zhang et al, 2005). Adicionalmente, o sistema atua como fonte constante de íons ferrosos, os quais poderiam fundamentar reaçóes posteriores do tipo Fenton (Tang et al, 1996). Além das reações de redução mediadas pela oxidação $\mathrm{Fe}^{0} / \mathrm{Fe}^{2+}$, outras duas vias redutivas podem ocorrer em solução aquosa. Uma em função da oxidação de íons ferrosos $\left(\mathrm{Fe}^{2+}\right)$ a férricos $\left(\mathrm{Fe}^{3+}\right)$ (Doong et al, 1998), e outra mediada por $\mathrm{H}_{2}$, que surge da corrosão do $\mathrm{Fe}^{0}$ pela água (Choe et al, 2004).

Por se tratar de um processo heterogêneo, é indispensável que as moléculas do contaminante entrem em contato com 
a superfície do ferro (Chen et al, 2001). Para corantes em meio ácido, entretanto, a reação ocorre preferencialmente por ataque da ligação azo pelo hidrogênio produzido, como já descrito por Feng et al (2000), a partir da oxidação do $\mathrm{Fe}^{0}$. O processo envolvendo ferro metálico pode ser empregado tanto para a remoção in situ de poluentes de águas subterrâneas (por exemplo, através de paredes permeáveis), como para o tratamento de efluentes contaminados (Westerhoff et al, 2003).

\section{OBJETIVOS}

O principal objetivo deste trabalho está representado pelo estudo da potencialidade da lã de aço comercial, como fonte de ferro metálico para a degradação redutiva de azo corantes, visando o desenvolvimento de processos contínuos orientados à remediação de resíduos líquidos oriundos do processo de beneficiamento têxtil.

\section{PARTE EXPERIMENTAL}

\section{Reagentes}

Os azo corantes Laranja Reativo 16 (C.I. 17757), Preto Reativo 5 (C.I. 20505) e Amarelo Brilhante 3G-P (C.I. 18972), todos da Aldrich, foram utilizados em solução aquosa de $100 \mathrm{mg} \mathrm{L}^{-1}$. As suas estruturas químicas são apresentadas na Figura 1. Outros reagentes utilizados foram de grau analítico PA.

Como fonte de ferro metálico foi utilizada lã de aço, disponível comercialmente em diversas gramaturas. As denominações "Tipo 1", "Tipo 2" e "Tipo 3" correspondem a fibras com diâmetros de 5, 11 e $10 \mathrm{~mm}$, respectivamente. A caracterização por espectrometria de fluorescência de raios-x (espectrômetro SHIMADZU, modelo EDX-700) permitiu estabelecer uma composição média constituída por ferro metálico (>98\%), manganês $(0,8 \%)$, enxofre $(0,1-0,2 \%)$ e carbono (0,1\%). Caracterizações microscópicas foram realizadas por microscopia eletrônica de varredura, utilizando-se equipamento JEOL (JSM-6360LV).

\section{METODLOGIA}

Amostras aquosas do corante foram bombeadas continuamente (vazão de aproximadamente $0,900 \mathrm{~L} \mathrm{~h}^{-1}$ ) para um reator cilíndrico de vidro $(26 \mathrm{~cm}$ de altura, $2,5 \mathrm{~cm}$ de diâmetro e volume útil de aproximadamente $100 \mathrm{~mL}$ ) recheado com lã de aço comercial (Figura 2). Parâmetros experimentais relevantes $(\mathrm{pH}$, massa e gramatura da lã de aço) foram otimizados utilizando-se sistemas de planejamento fatorial de experimentos. Amostras foram coletadas a cada $30 \mathrm{~min}$ na saída do reator, sendo submetidas a controle analítico.

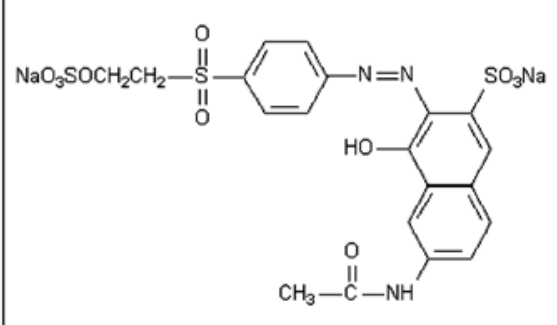

A<smiles>CS(=O)(=O)OCCS(=O)(=O)c1ccc(N=Nc2c(S(=O)(=O)O)cc3cc([S+]([O])(=O)O[Na])c(N=Nc4ccc(S(=O)(=O)CCS(=O)(=O)O[Na])cc4)c(O)c3c2N)cc1</smiles>

B<smiles>Cc1nn(-c2cc(Cl)c(O[Na])cc2Cl)c(O)c1N=Nc1cc(Nc2nc(Cl)nc(Nc3ccc(O[N+](=O)[O-])cc3)n2)ccc1[N+](=O)[O-]</smiles>

C

Figura I- Estruturas químicas dos corantes laranja reativo I 6 (A), preto reativo 5 (B) e amarelo brilhante 3G-P (C)

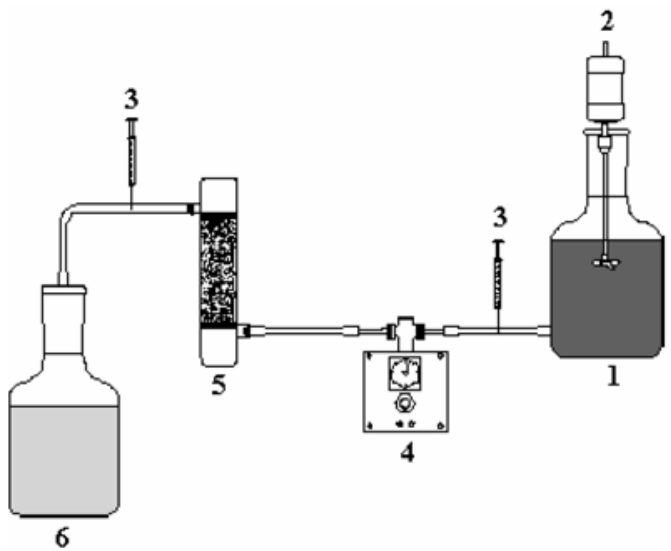

1 - Recipiente de amostra (2 L);

2 - Sistema de agitação mecânica;

3 - Pontos de coleta de amostra;

4 - Bomba peristáltica;

5 - Coluna recheada com lã de aço;

6 - Recipiente de amostra tratada.

Figura 2 - Representação esquemática do reator 


\section{Controle analítico}

O processo de degradação redutiva do corante foi acompanhado por espectroscopia UV-VIS (espectrofotômetro S-1150 HP), monitorando a redução da área espectral entre 350 e $700 \mathrm{~nm}$. O resultado é expresso na forma do quociente entreárea final eárea inicial $\left(\mathrm{A} / \mathrm{A}_{0}\right)$.

A evolução de íons ferro foi acompanhada espectrofotometricamente, por meio de metodologia fundamentada na reação entre $\mathrm{Fe}^{2+}$ e o-fenantrolina.

Determinações de Carbono Orgânico Total (COT) foram realizadas em um analisador de carbono (TOC-5000ASHIMADZU).

\section{RESULTADOS E DISCUSSÃO}

\section{Estudos preliminares}

Com o objetivo de verificar a potencialidade da utilização de ferro metálico na degradação de soluções aquosas contendo azo-corantes, vários estudos preliminares foram realizados. Nestes estudos ficou claramente demonstrada a potencialidade da lá de aço comercial, como fonte de ferro metálico, assim como foi possível identificar variáveis operacionais de importância, cujo efeito foi posteriormente estudado por meio de sistemas de planejamento fatorial de experimentos (Barros Neto et al, 2002). Nestes estudos, o efeito das variáveis $\mathrm{pH}$, massa e espessura da fibra de aço foram estudados em dois níveis, o que configura um planejamento fatorial $2^{3}$, acrescido de um ponto central ensaiado em sextuplicata (Tabela 1). Uma vez que o sistema proposto visa a degradação de corantes, a resposta utilizada foi à evolução da área espectral registrada entre 350 e $700 \mathrm{~nm}$, expressa na forma de um quociente entre área final e inicial $\left(\mathrm{A} / \mathrm{A}_{0}\right)$. Adicionalmente, foi monitorado o teor de ferro II liberado durante o processo, de maneira a verificar condiçôes que permitam um máximo de degradação, acompanhado de liberação de ferro compatível com os limites impostos pela legislação vigente (Resolução CONAMA 357 de 2005), que estabelece em $15 \mathrm{mg} \mathrm{L}^{-1} \mathrm{o}$ valor máximo de ferro solúvel para lançamento de resíduos em corpos de água.

De acordo com os resultados apresentados na Tabela 1 , e levando-se em consideração os efeitos calculados a partir deles, verifica-se que as maiores taxas de degradação são viabilizadas por uma maior massa do material, constituído por fibras de menor espessura (Tipo 1). Trata-se de um resultado esperado, coerente com os antecedentes reportados na literatura, principalmente levando-se em consideração o caráter heterogêneo do processo. Por outro lado, o efeito do $\mathrm{pH}$ é bastante discreto, confundindo-se com o desvio padrão típico observado na análise em sextuplicata $(0,020)$. Esta última observação é contrária ao normalmente exposto na literatura, onde normalmente associa-se uma maior eficiência de degradação a baixos valores de $\mathrm{pH}$. Entretanto, uma comparação entre os resultados do experimento 3 e 7 evidenciam a existência de poucas diferenças na eficiência de degradação em meio ácido e neutro.

Uma vez que a eficiência de degradação não é significativamente sacrificada em $\mathrm{pH}$ neutro, ao passo que a liberação de íons ferrosos pode ser bastante diminuída, as condiçôes do experimento 7 foram selecionadas para estudos posteriores.

\section{Degradação de corante modelo}

Operando o sistema nas condições previamente otimizadas, e monitorando o processo por espectroscopia UV-Vis, é possível observar que o tempo de retenção de aproximadamente 6 min é suficiente para degradar os cromóforos responsáveis pela forte absorção observada entre 350 e 700 nm (Figura 3). As estruturas aromáticas, que absorvem fortemente na região próxima a $300 \mathrm{~nm}$, são igualmente degradadas com eficiência, restando apenas um sinal residual atribuído ao íon ferroso e aos subprodutos do processo de degradação.

Tabela I-Resultados da otimização obtidos através de planejamento fatorial $2^{3}$. Substrato: corante preto reativo $5\left(100 \mathrm{mg} \mathrm{L}^{-1}\right)$

\begin{tabular}{cccccc}
\hline Experimento & \multicolumn{3}{c}{ Variáveis } & \multicolumn{2}{c}{ Respostas } \\
& Tipo de Fe Massa de Fe (g) & $\mathrm{pH}$ & $\mathrm{A}_{0} \mathrm{~A}_{0}$ & $\mathrm{Fe}^{2+}\left(\mathrm{mg} \mathrm{L}^{-1}\right)$ \\
\hline 1 & 1 & 3 & 3 & 0,064 & 7,1 \\
2 & 3 & 3 & 3 & 0,300 & 28,3 \\
3 & 1 & 10 & 3 & 0,015 & 8,7 \\
4 & 3 & 10 & 3 & 0,054 & 33,4 \\
5 & 1 & 3 & 7 & 0,076 & 1,0 \\
6 & 3 & 3 & 7 & 0,340 & 2,5 \\
7 & 1 & 10 & 7 & 0,037 & 1,1 \\
8 & 3 & 10 & 7 & 0,062 & 12,8 \\
Ponto central & 2 & 6 & 5 & $0,080+/-0,020$ & $2,4+/-3,2$ \\
\hline
\end{tabular}

Efeitos principais para a resposta $\mathrm{A} / \mathrm{A}_{0}$ :

Tipo de Fe: 0,141 +/- 0,020; Massa de Fe: -0,153 +/- 0,020; pH: 0,021 +/- 0,020

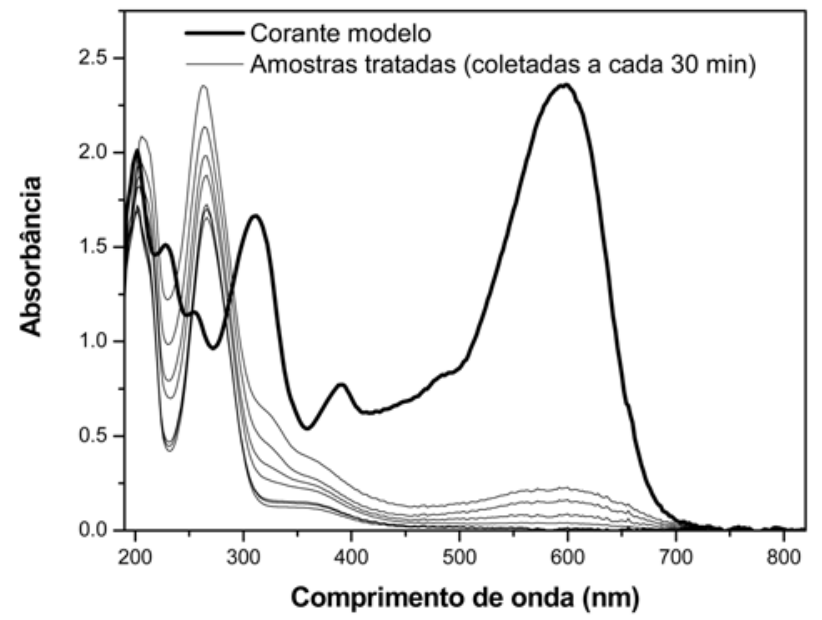

Figura 3 - Monitoramento espectrofotométrico do processo de degradação em pH 7

Substrato: corante preto reativo 5 (100 $\left.\mathrm{mg} \mathrm{L}^{-1}\right)$ 
A mineralização efetiva do substrato foi avaliada monitorando-se o teor de carbono orgânico total (COT). Os resultados (não apresentados) indicam uma eficiência inicial da ordem de $50 \%$, eficiência esta que é reduzida a praticamente a metade durante o transcurso de $5 \mathrm{~h}$ de tratamento contínuo. A diminuição da eficiência provavelmente está relacionada com a passivação do ferro metálico por deposição de óxidos, fator este que pode ser contornado pela lavagem com soluçôes levemente aciduladas, a cada 8 horas de uso. A corrosão das fibras de aço como resultado da sua utilização no processo pode ser visualizada nas micrografias eletrônicas apresentadas na Figura 4 (aumento de 5000 vezes). Trata-se de um efeito do qual depende a eficiência do processo redutivo, efeito este que faz com que a substituição das fibras deva ser considerada após aproximadamente 50 horas de uso contínuo.

\section{Degradação de mistura de corantes}

Utilizando-se as mesmas condiçōes experimentais descritas anteriormente, a capacidade do sistema redutivo foi avaliada frente a soluções aquosas contendo uma mistura de 3 corantes reativos. Os resultados (Figura 5) confirmam uma elevada eficiência do sistema, principalmente em relação à degradação dos grupos cromóforos que caracterizam as moléculas de corante. Uma significativa redução do sinal espectral pode ser observada na região centrada em $300 \mathrm{~nm}$, o que sugere uma eficiente degradação de estruturas aromáticas, também bcaracterísticas dos substratos em estudo.

\section{CONCLUSÕES}

O sistema descrito nesse trabalho se apresenta como uma alternativa promis- sora para tratamento de corantes têxteis, principalmente em razão da simplicidade operacional e do baixo custo dos insumos envolvidos.

Embora os resíduos do processo de tingimento de fibras têxteis apresentem uma composição mais complexa, principalmente em função da presença de agentes químicos auxiliares, os resultados sugerem uma elevada potencialidade do sistema na remediação contínua de resíduos desta natureza.

De maneira geral, a utilização de um sistema contínuo fundamentado no uso de lã de aço permite eficiente degradação das moléculas de corante, o que redunda em uma completa remoção da cor. Levando-se em consideração a extrema resistência destes substratos frente a processos biológicos convencionais, os resultados se mostram extremamente promissores.

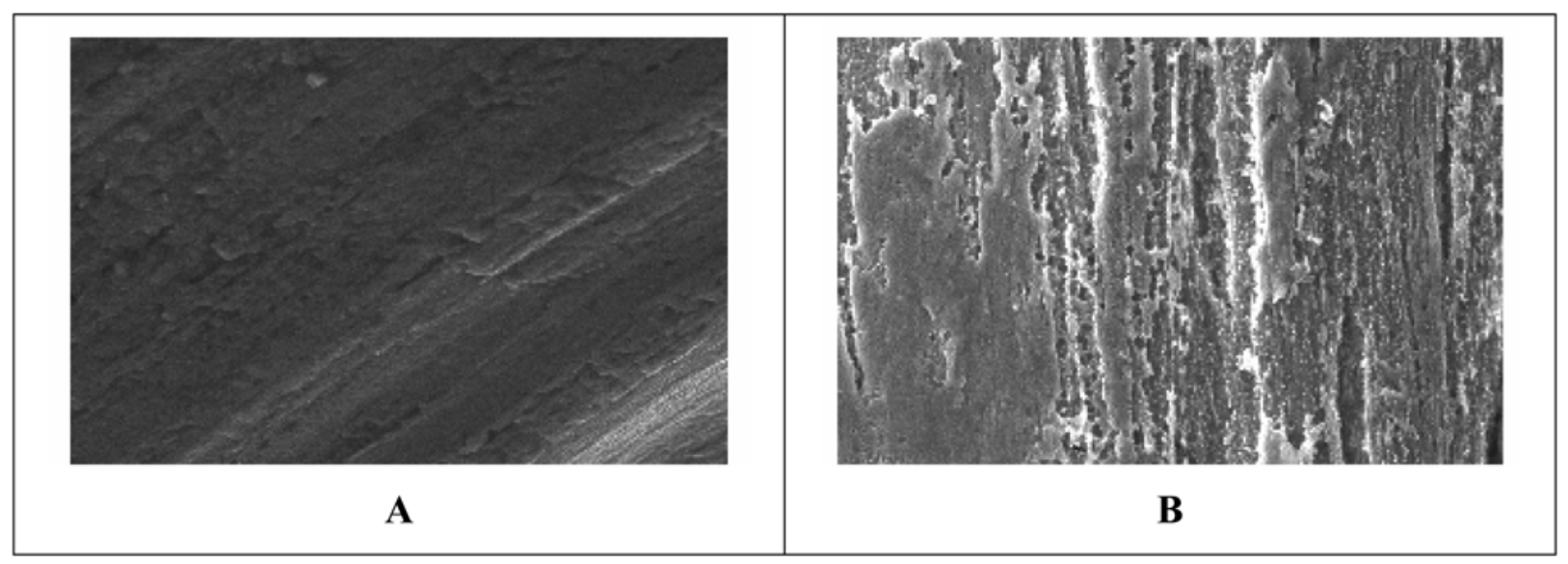

Figura 4 - Micrografias eletrônicas de fibras novas (A) e usadas (B)

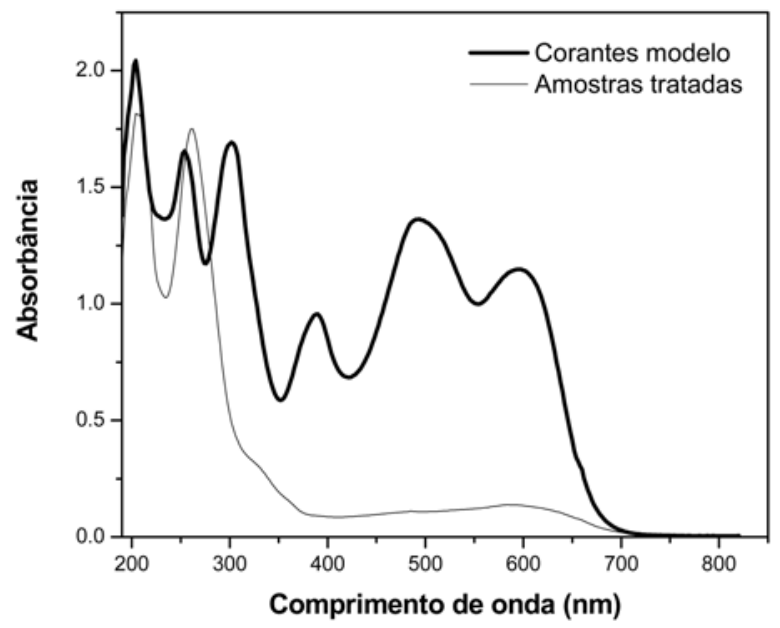

Figura 5 - Monitoramento espectrofotométrico do processo de degradação em pH 7.

Substrato: mistura de corantes reativos (33,3 $\mathrm{mg} \mathrm{L}^{-1}$ cada um) 


\section{REFERÊNCIAS}

BARROS NETO, B; SPACINO, I. S. BRUNS, R. E. Como fazer experimentos, ed. Campinas, Ed. Unicamp, 2002.

CHEN, J. L. et al. Effects of $p H$ on dechlorination of trichloroethylene by zero-valent iron. Journal of Hazardous Materials, v. B83, p. 243-254, 2001.

CHOE, S.; LILJESTRAND, H. M.; KHIM,

J. Nitrate reduction by zero-valent iron under diferent $p H$ regimes, Applied Geochemistry, n.19, p. 335-342, 2004

DOONG, R.; CHANG, W. Photodegradation of parathion in aqueous titanium dioxide and zero valent iron solutions in the presence of hydrogen peroxide. Journal of Photochemistry and Photobiology A: Chemistry, v.116, p. 221228, 1998 .

FENG, W.; NANSHENG, D.; HELIN, $\mathrm{H}$ Degradation mechanism of azo dye C.I. reactive red 2 by iron powder reduction and photooxidation in aqueous solutions. Chemosphere, v. 41 p. 1233-1238, 2000.
GUARATINI C. C. I.; ZANONI M.V. B. Corantes têxteis. Química nova, v. 23, n.1, p. 71-78, 2000.

PEREY, J. R. et al. Zero-valent Iron Pretreatment for Enhancing the Biodegradability of Azo dyes. Water Environment Research, v. 74, n. 3, p. 221-225, May/June. 2002

TANG, W.Z.; CHEN, R.Z. Descolorization kinetics and mechanisms of commercial dyes by $\mathrm{H}_{2} \mathrm{O}$ /iron powder system. Chemosphere, v. 32, n.5, p. 947-958, 1996.

TOLEDO, R. A. S. Visão do mercado têxtil. Química têxtil, n. 72, p. 8-10, 2003.

WESTERHOFF, P.; JAMES, J.. Nitrate removal in zero-valent iron packed columns. Water Research, v. 37, p. 1818-1830, 2003.

ZHANG H. et al. The use of ultrasound to enhance the decolorization of the C.I. Acid Orange 7 by zero-valent iron. Dyes and Pigments, v. 65, p. $39-45,2005$
Endereço para correspondência:

Patricio Peralta-Zamora

Departamento de Química

Universidade Federal do Paraná

Caixa Postal I908I

8I53 I-990 - Curitiba - PR - Brasil

E-mail: zamora@quimica.ufpr.br

\section{Revisto Engenhoria Sonitória e Ambientol}

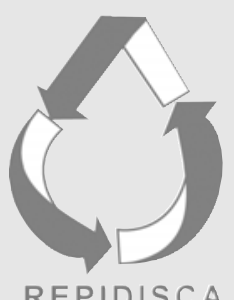

Repidisca - Rede Panamericana de I nformaciones en Salud Ambiental

http/ / www.cepis.org.pe

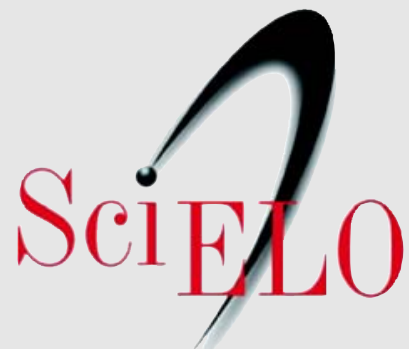

\section{Scientific Electronic Library Online}

http:/ / www.scielo.org 